\title{
POLÍTICAS DE DESARROLLO EN EL MEDIO RURAL: UNA REVISIÓN DE LA LEGISLACIÓN DE LA UNIÓN EUROPEA
}

\author{
M. LAGUNA MARÍN-YASELI \\ Instituto Pirenaico de Ecología (CSIC, Zaragoza)
}

El documento europeo conocido como Agenda 2000, ratificado en el Acuerdo de Berlín y donde se recogen las perspectivas generales de desarrollo de la Unión Europea, reconocía que no existe una política de Desarrollo Rural y que ésta es una yuxtaposición de políticas de mercados agrarios, política estructural y medioambiental (PARLAMENTO EUROPEO, 1998) ${ }^{1}$. Esta afirmación, siendo cierta, no recoge la complejidad global del problema. La Agenda 2000, al revisar el concepto de política rural, se centra en el apartado dedicado a la reforma de la Política Agraria Comunitaria (PAC), olvidando que lo rural hace sobre todo referencia a una concepción particular del espacio que tiene su reflejo en la ordenación del territorio (LÓPEZ GÓMEZ Y ARROYO ILERA, 1987) y que no es meramente agrario. Este hecho remite por un lado a la política territorial o regional, y por otro no puede olvidar las acciones derivadas del uso que el hombre hace de ese espacio (política agraria, de transportes, turismo, infraestructuras, industria...).

Esta superposición de políticas viene dada por la propia concepción del Desarrollo Rural como un conjunto complejo, integral, sistémico y global (VALCÁRCEL-RESALT, 1989; ATIENZA 1992; CEÑA 1994; GóMEZ OREA, 1999). El objetivo de este trabajo es sintetizar la normativa establecida por la Unión Europea para realizar una política de Desarrollo Rural, dedicando especial atención a la referida a las áreas de montaña.

\section{LA POLÍTICA AGRARIA.}

Una de las principales bases del Desarrollo Rural es la política agraria. Las

1 “La política rural en la Unión Europea se presenta todavía como una yuxtaposición de la política de mercados agrarios, la política estructural y la política medioambiental, con instrumentos bastante complejos y con una falta de coherencia global" (COM(97) 
zonas rurales se basaban tradicionalmente en la actividad primaria. Esta situación ha sido modificada recientemente; uno de los principales objetivos del Desarrollo Rural actual es la búsqueda de fuentes de ingresos alternativas a esta actividad. No obstante, las actuaciones de la política agraria han dejado una profunda huella en el mundo rural y siguen teniendo una vigencia bastante considerable. En este sentido, en la Agenda 2000 se reconoce al Desarrollo Rural como el segundo pilar de la PAC tras la política de precios y mercados. Las actividades tradicionales como la agricultura y la ganadería cumplen una función especialmente relevante en las zonas de montaña, evitando la matorralización y la pérdida de recursos herbáceos (VICENTE SERRANO, 2000).

En el Tratado de Roma (1957) ya se concedía especial importancia al sector agrario, considerado como una actividad estratégica (MAYA Y CABERO, 2000). No hay que olvidar que Francia y Alemania son socios fundadores con gran peso de la actividad agraria en su economía. En 1962 se instauraba la Política Agraria Comunitaria (PAC), basada en tres principios fundamentales: Unidad de mercado, preferencia comunitaria y solidaridad financiera. Estos principios se establecían para alcanzar varios objetivos: Incremento de la productividad agrícola, desarrollo racional de la producción agraria, empleo óptimo de los factores de producción, abastecimiento de mercados, garantía de un nivel de vida equitativo a los agricultores y asegurar a los consumidores precios asequibles. Dichos objetivos se mantuvieron sin variación hasta 1992, año en que los problemas excedentarios y medioambientales contribuyeron a acelerar la reforma de esta política.

La PAC desde su inicio nace con dos orientaciones fundamentales, la política de mercados y la política de estructuras.

\section{a) Política de precios y mercados}

El artículo 38 del Tratado de Roma es la base jurídica de la política de precios y mercados. De él se derivan los reglamentos base que rigen las distintas Organizaciones Comunes de Mercado (OCMs):

1. El mercado común abarcará la agricultura y el comercio de los productos agrícolas. Por productos agrícolas se entienden los productos de la tierra, de la ganadería y de la pesca, así como los productos de primera trans-

2000 final, Vol. I, Agenda 2000. Por una Unión más fuerte y más amplia).

Polígonos. Revista de Geografía, 11-12 (2001-2002); pp. 219-233. 
formación directamente relacionados con aquéllos.

2. Salvo disposición en contrario de los artículos 39 a 46, ambos inclusive, las normas previstas para el establecimiento del mercado común serán aplicables a los productos agrícolas.

Las OCMs son los instrumentos básicos para orientar la producción agrícola y estabilizar los mercados. Se fueron regulando paulatinamente, y en 1994 cubrían casi todos los productos agrícolas (90\% de la Producción Final Agraria de la Unión) (Parlamento Europeo, 1998). Existen 5 tipos de OCMs:

- OCMs con precios de garantía y ayudas directas complementarias a la producción: vacuno, ovino, cereales, arroz...

- OCMs de intervención automática: azúcar, leche y lácteos

- OCMs de intervención condicionada: vino, porcino...

- OCMs sólo de ayudas directas a la producción: oleaginosas, proteaginosas, cereales...

- OCMs sin apoyo directo a la producción que sólo gozan de protección aduanera: aves, huevos, algunas hortalizas y frutas

En lo que se refiere a las áreas de montaña, son dos las OCMs que interesan especialmente por tener un complemento para zonas desfavorecidas de montaña; la OCM de carne de vacuno y la OCM de carne de ovino y caprino.

\section{b) Política de estructuras}

La formulación global de la política de estructuras se estableció en el conocido como Plan Mansholt (1968), que tenía como objetivos el gestionar el proceso de ajuste estructural, la modernización de las explotaciones eficientes y la reducción de los costes sociales. Siguiendo esta pauta en 1972 se formularon los principios rectores de las tres primeras directivas socioestructurales: incentivos a las inversiones de modernización en las explotaciones (72/159/CEE), cese de la actividad agrícola y reasignación de la tierra con fines de mejora estructural (72/190/CEE) y cualificación y orientación profesional de la población agrícola (72/161/CEE). En 1975 y dentro de esta política estructural, se gestó "el primer elemento de la política conservacionista del Desarrollo Rural" (PARLAMENTO EUROPEO, 1998; ORDÓÑEZ, 1999); se trata de la Directiva 268/75/CEE sobre zonas desfavorecidas y de montaña. En ella se reconoce que no todas las agriculturas de la Comunidad tienen los mismos problemas, que no existe un mode- 
lo único de agricultura y que hay zonas con deficiencias específicas derivadas de su medio físico o de la escasez de población. Se introduce por primera vez los pagos por medio de ayudas directas que serán totalmente implantados tras la reforma de 1992. Este pago directo es la Indemnización Compensatoria (IC), prima única anual para compensar a los titulares de explotación por las pérdidas derivadas de los problemas del medio físico y las carencias del medio humano.

En la década de 1980 la política de estructuras va a sufrir una serie de alteraciones profundas, motivadas por la crisis del sector que presenta altas tasas de desempleo, transformaciones en los mercados internacionales de productos agrarios, excedentes, presiones del GATT y problemas medioambientales. El rumbo de la política de estructuras se dirige a la mejora de las explotaciones y a la gestión medioambiental. Además se asiste a la introducción del sistema de cuotas y al inicio del enfoque rural (MAYA Y CABERO, 2000).

El reglamento 797/85 relativo a la Mejora de Estructuras Agrarias desarrolla varias acciones a favor de las explotaciones persiguiendo objetivos concretos como mantenimiento de la población, mejora de las condiciones de trabajo, vida e higiene y protección del medio ambiente. Estas acciones se han mantenido sin cambios significativos hasta la actualidad por medio de los reglamentos de mejora de estructuras agrarias (Reglamentos 2328/91, 950/97 y de desarrollo rural 1257/99).

No obstante, no será hasta la reforma de 1992 cuando los problemas estructurales gocen de un papel destacado. De hecho, MAYA Y CABERO (2000) han señalado que en la reforma de 1992 se introducen nuevos capítulos en la PAC. Así, a la función productivista de la agricultura se le une la conservacionista y se trabaja en la concepción del Desarrollo Rural como algo no meramente agrario, en el control de las producciones agrarias y en el fomento de las medidas de acompañamiento.

Las reformas llevadas a cabo en 1992 se resumen en el denominado decálogo MacSharry (RAMOS Y ROMERO, 1995):

- Mantener población

- Relacionar la producción con la protección medioambiental

- Diversificar la economía

- Controlar los excedentes y el gasto 
- Mantener los precios con fórmulas diferentes hasta entonces

- Recoger producción de calidad y respetuosa con el medio ambiente

- Reafirmar los principios originarios de la PAC

- Incrementar las medidas de estabilización

- Solidaridad financiera

- Asumir la creciente interdependencia nacional

Como afirma el Parlamento Europeo (1998), esta reforma supone un cambio radical puesto que en el proceso de reducción de excedentes y precios se introducen las ayudas directas por hectárea o cabeza de ganado dentro de las Organizaciones Comunes de Mercado.

Entre las acciones incluídas en la reforma de 1992 se destacan la puesta en marcha de las medidas de acompañamiento: Agroambientales (R 2078/92), Cese anticipado de la actividad agraria (2079/92) y Medidas Forestales (2080/92). $\mathrm{Su}$ financiación supone un cambio en el sistema de pagos efectuado hasta ese momento. Si las anteriores medidas (excepto la regulación de precios de las OCMs) eran financiadas por la sección orientación del Fondo Estructural de Orientación y Garantía Agrarias (FEOGA-O), las medidas de acompañamiento van a depender de la sección Garantía de FEOGA (FEOGA-G). Este aumento de la importancia del FEOGA-G en la financiación del desarrollo rural se va a mantener hasta la Agenda 2000, donde el FEOGA-O se dedicará a parte de las iniciativas comunitarias y de las acciones de Desarrollo Rural dentro del Objetivo 1. Por su parte, el FEOGA-G se ocupará del resto de las medidas y acciones encaminadas a la consecución de este desarrollo.

La Agenda 2000 sienta las bases de la nueva política de Desarrollo Rural dentro de la PAC. Así, el objetivo de la reforma de la PAC y el apoyo al Desarrollo Rural según este documento es: "Implantar una política integrada de Desarrollo Rural Sostenible por medio de un instrumento jurídico único que garantice una mayor coherencia entre el Desarrollo Rural y la política de precios y mercados de la Política Agraria Común (PAC), y potenciar todos los elementos integrantes del Desarrollo Rural fomentando la participación de los agentes locales"

Esta nueva concepción del Desarrollo Rural se transforma en el segundo pilar de la Política Agraria Común (COMISIÓN EUROPEA, 1999), aunque muy por de- 
bajo del presupuesto asignado a precios y mercados (ver tabla 1).

Tabla 1. PAC; gastos sección garantía periodo 2000-2006 (en miles de euros)

\begin{tabular}{lrrrrrrrr}
\hline & 2000 & 2001 & 2002 & 2003 & 2004 & 2005 & 2006 & Total \\
\hline Total PAC & 40,92 & 42,80 & 43,90 & 43,77 & 42,76 & 41,93 & 41,66 & 297,74 \\
Mercados & 36,62 & 38,48 & 39,57 & 39,43 & 38,41 & 37,57 & 37,29 & 267,37 \\
Desarrollo rural & 4,30 & 4,32 & 4,33 & 4,37 & 4,35 & 4,36 & 4,37 & 30,37 \\
\hline
\end{tabular}

FUENTE: COMISIÓN EUROPEA, 1999

En la tabla 1 se manifiesta que el presupuesto de la PAC aumenta hasta 2003 para ir perdiendo progresivamente importancia hasta 2006, año en el que el presupuesto de la PAC es 1,8\% superior a 2000. La política de precios y mercados aumenta el mismo porcentaje mientras que el Desarrollo Rural lo hace en 1,6\%.

Las acciones a favor del Desarrollo Rural se recogen en el Reglamento 1257/99 (modificado por los reglamentos 1452/2001; 1453/2001 y 1454/2001) desarrollado por el R 1750/99 (modificado por los reglamentos 2075/2000; $672 / 2001$ y 1763/2001). Las acciones de desarrollo rural se pueden agrupar en dos grandes grupos; por un lado, las medidas introducidas en la reforma de 1992 (cese anticipado, medidas agroambientales y medidas forestales), junto con las ayudas a las zonas desfavorecidas y, por otro, las encaminadas a la modernización y diversificación de la actividad agraria (inversiones en las explotaciones, instalación de jóvenes agricultores, formación profesional, mejora de la producción agraria y fomento de las zonas rurales). Este reglamento engloba las acciones que antes se dispersaban en la Directiva sobre zonas de montaña, en los reglamentos de mejora de las estructuras agrarias y en los relativos a las medidas de acompañamiento. Aunque, como se ha mencionado con anterioridad, la política agraria es tan sólo una de las manifestaciones de este Desarrollo Rural. Es necesario atender a otros factores como la política regional, el turismo y la industria.

\section{POLÍTICA REGIONAL.}

En el Tratado de Roma no se contemplaba el establecimiento de una política regional comunitaria, aunque sí que se manifestaba la necesidad de una reducción de las desigualdades entre las diferentes regiones de Europa. En 1973, coincidiendo con la primera ampliación de la Comunidad, se asiste al nacimiento de esta política (El Fondo Europeo de Desarrollo Regional- FEDER- fue creado 
en 1975). El objetivo de paliar las diferencias regionales, tiene un reconocimiento expreso para las zonas rurales en el artículo 130 A del Tratado de Maastrich: "La comunidad se propondrá, en particular, reducir las diferencias entre los niveles de desarrollo de las diversas regiones y el retraso de las regiones más desfavorecidas, incluidas las zonas rurales".

El elemento fundamental de actuación de la política regional son los Fondos Estructurales, creados como instrumentos de solidaridad financiera y reformados en 1988 por el Reglamento 2052/88. Los Fondos Estructurales (FEOGA, Fondo Europeo de Desarrollo Regional- FEDER- y Fondo Social EuropeoFSE-) actuaban hasta la reforma de la Agenda 2000 sobre cinco objetivos prioritarios:

- Objetivo 1: Desarrollo y ajuste estructural de las regiones menos desarrolladas FEDER, FSE, FEOGA

- Objetivo 2: Reconversión de las regiones en declive industrial FEDER, FSE

- Objetivo 3: Lucha contra el paro de larga duración FSE

- Objetivo 4: Inserción profesional de los Jóvenes FSE

- Objetivo 5:

- Acelerar la adaptación de las estructuras agrarias FEOGA-O

- Desarrollo de las zonas rurales FEOGA-O, FEDER, FSE

Con la entrada de Austria, Suecia y Finlandia en 1994 en la Unión Europea se estableció un nuevo objetivo (6) relativo a los problemas específicos de las regiones con muy débil densidad de población.

Cada uno de estos objetivos cuenta con un Marco Comunitario de Apoyo (MCA) que incluye los ejes prioritarios de actuación, el plan de acción y el suministro de medios financieros. Los Programas Operativos (PO) concretan las acciones y medidas a desarrollar, con subprogramas para cada fondo (cuadro 1).

Cada MCA se concreta en un Programa Operativo (PO) que se desarrolla mediante subprogramas para cada Fondo 
Cuadro 1: Participación de las diferentes administraciones en la elaboración de los MCA y PO para cada objetivo comunitario

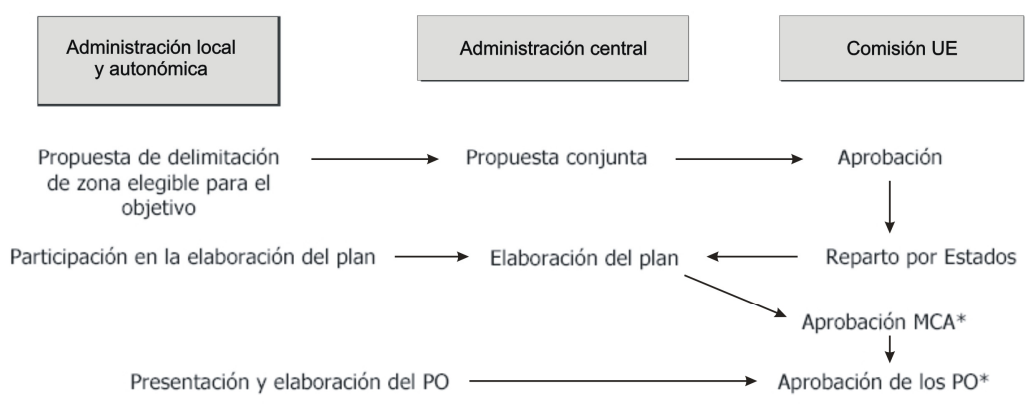

FUENTE: PUJADAS Y FONT (1998)

* En 1993 el Consejo aprobó una medida mediante la cual los Estados Miembros podían presentar un único documento (conocido como DOCUP, Documento Único de Programación que recogiese un plan de desarrollo y la solicitud de intervención de los Fondos Estructurales).

Los fondos estructurales FEDER, FEOGA-O y FSE se regulaban tras la reforma de 1988 por los reglamentos 4254/88, 4255/88 y 4256/88. En esta reforma se introdujeron también las Iniciativas Comunitarias, instrumento específico de la política regional (CHAMPETIER, 1996; USERO, 1998). La reforma de 1988 estuvo motivada por la elaboración del Acta Única Europea (1986). Los principios de dicha reforma pueden resumirse en cuatro grandes apartados: concentración de fondos por objetivo y región, colaboración entre la Comisión, Estados Miembros y autoridades regionales para la planificación, establecimiento y seguimiento de las intervenciones, programación de las intervenciones y adicionalidad de la contribución comunitaria (PARLAMENTO EUROPEO, 2001).

Tras la firma del Tratado de Maastrich en 1992 se planteó la necesidad de una nueva reforma de los fondos estructurales, y se asistía a la creación del Fondo de Cohesión. En esta nueva reforma, regida por los Reglamentos 2081/93 (modifica 2052/88) y 2082/93 (modifica 4253/88), se incluyeron las siguientes modificaciones:

- Adaptación de los objetivos prioritarios de los fondos a los cambios eco- 
nómicos en curso y revisión del Fondo Social Europeo.

- Revisión del procedimiento de establecimiento de los objetivos 2 y 5 b.

- Simplificación de los procedimientos de programación.

- Consolidación de la colaboración de los interlocutores económicos y sociales.

- Intensificación de las evaluaciones (ex ante, seguimiento y ex post).

- Importancia del principio de adicionalidad y sostenibilidad.

- Fomento de la igualdad entre hombres y mujeres.

- Mayor participación del Parlamento Europeo en la aplicación de las políticas estructurales.

En esta reforma se adoptaron de nuevo las iniciativas comunitarias. De las trece $^{2}$ que se desarrollaron en el conjunto de la Unión una interesa especialmente en el Desarrollo Rural de las áreas de montaña: LEADER.

La Iniciativa comunitaria LEADER (Liason Entre Activitées de Developpement de l'Economie Rurale) tiene como objetivo principal la movilización de la población rural para que ésta sea artífice de su propio desarrollo (CHAMPETIER, 1996). Con un enfoque de abajo hacia arriba (conocido como "bottom-up"), impulsa la participación de los diferentes agentes rurales. Desde su puesta en marcha en 1991 ha habido dos ediciones LEADER I (1991-1993) y LEADER II (1994-1999) y ya se está desarrollando el LEADER+ (2000-2006).

Recientemente, a raíz de la Agenda 2000, han sido modificados los objetivos y Fondos Estructurales Comunitarios por los reglamentos 1260/99 (modificado por el Reglamento 1447/2001). El objetivo 1 agrupa a las regiones que sufren un retraso en su desarrollo. Son regiones cuyo PIB por habitante es menor al $75 \%$ de la media comunitaria, regiones ultraperiféricas, muy poco pobladas de Finlandia y Suecia que anteriormente pertenecían al objetivo 6. El objetivo 2 recoge las zonas que manifiestan problemas específicos de reconversión económica y estructural. Son las zonas rurales sensibles, zonas en las que los secto-

\footnotetext{
${ }^{2}$ Los ámbitos de aplicación de estas iniciativas definidos en 1993 son los siguientes: cooperación interregional (INTERREG, REGEN), empleo y mano de obra (EUROFORM, Now, HORIzON), desarrollo industrial (RECHAR, RESIDER, PRISMA, TELEMATICA y STRIDE), regiones ultraperiféricas (REGIS), política urbana (URBAN) y desarrollo rural (LEADER).
} 
res de la industria y los servicios están registrando cambios económicos y sociales, zonas urbanas en crisis y zonas dependientes del sector pesquero. Por último, el objetivo 3 es un objetivo de tipo horizontal que se destina a la adaptación y modernización de las políticas y sistemas de educación, formación y empleo de las zonas no cubiertas por los objetivos 1 y 2 . También se producen en esta reforma modificaciones en la financiación de la Política Agraria Común. Tal y como se avanzó en el apartado anterior, la sección Garantía del FEOGA cobra un mayor protagonismo. La tabla 2 anota las fuentes de financiación antes y después de 2000. Las Indemnizaciones Compensatorias son ahora financiadas por la sección garantía, así como la totalidad de las acciones emprendidas en el objetivo 2. La sección orientación queda relegada a las zonas de objetivo 1 (salvo las medidas de acompañamiento) y a la financiación de la iniciativa LEADER.

Tabla 2. Fuentes de financiación antes y después de 2000

\begin{tabular}{lcc}
\hline & $1994-1999$ & $2000-2006$ \\
\hline Medidas de acompañamiento & Garantía (IC sin incluir) & Garantía (IC incluídas) \\
Indemnizaciones compensatorias & Orientación & Garantía \\
Objetivo 1 & Orientación & Orientación \\
Objetivo 2 (nuevo) & Garantía \\
Antiguo objetivo 5A & Orientación & - \\
Antiguo objetivo 5B & Orientación & - \\
Iniciativa LEADER & Orientación & Orientación \\
\hline
\end{tabular}

Fuente: Comisión Europea, 1999

Las iniciativas comunitarias también presentan cambios sustanciales. Se reducen de 13 a 4, manteniéndose la que resultaba más interesantes en la etapa anterior para el desarrollo rural, LEADER+ .

\section{OTRAS POLÍTICAS: TURISMO.}

El Desarrollo Rural dentro de la Unión Europea tiene, como ya se ha mencionado, dos orientaciones fundamentales: la política agraria y regional. Sin embargo, sobre el territorio rural de la Unión Europea se implementan muchas otras actividades económicas como la industria, la artesanía, la prestación de servicios o el turismo. De entre todas ellas, el turismo es la que está jugando un papel muy importante en la reestructuración de las regiones de montaña 
(ZIMMERMAN, 1994). Sin embargo, en relación con el turismo rural no existe en la Unión Europea una política concreta. El tratado de la CE no autoriza a la Comunidad a efectuar una política propia en materia turística, aunque si se le permite adoptar en el marco de otras políticas medidas de orientación y desarrollo del sector (PARLAMENTO EUROPEO, 2001). En esta línea se han desarrollado diferentes documentos de trabajo y disposiciones de organismos europeos como la Comisión, El Comité de las Regiones o el Parlamento Europeo. Entre ellos destacan documentos como Medidas comunitarias para el fomento del Turismo Rural (COM 90/438) y el Dictamen sobre una política de desarrollo del turismo rural en las regiones de la Unión Europea, elaborados por la Comisión y el Comité de las Regiones respectivamente.

En el primero de ellos se intentaba establecer un marco de coordinación entre las diferentes medidas de fomento de esta actividad, que tenían por objeto la definición de los productos de turismo rural, la mejora de la oferta, su promoción y comercialización.

En el segundo se insiste en la importancia del turismo rural como eje de una política rural y su papel como factor complementario en una estrategia de desarrollo regional. El turismo rural está necesitado de un enfoque global sustentado en cinco principios directores: conocer, ordenar, organizar, formar y gestionar. El informe concluye con la afirmación de la inexistencia en el seno de la Unión de competencias en el ámbito del turismo y de la participación de diferentes políticas (agraria y regional principalmente) en la promoción de este turismo.

No obstante, han sido los Fondos Estructurales a través de las Iniciativas Comunitarias (especialmente LEADER) los que han contribuído de forma más activa en el desarrollo de un turismo rural. En la primera edición del LEADER (1991-1993), el turismo rural fue la medida más importante en términos financieros, con una inversión de 490 millones de ecus (42\% del total). Las medidas llevadas a cabo se encontraban inmersas en los siguientes tipos de acciones: inversiones individuales o colectivas, adaptación de infraestructuras públicas a necesidades de la oferta, medidas de ampliación del periodo turístico, inventarios turísticos, estudios de mercado y actividades de promoción, e introducción de sistemas de reserva.

En el periodo 1994-1999 (LEADER II) las medidas desarrolladas incluyen las de la edición anterior y a ellas se le añade una acción específica encaminada a la conceptualización de aplicación de nuevos productos de Turismo Rural (inclu- 
yendo el Turismo Cultural y de Descubrimiento del Medio Ambiente).

\section{DESARROLLO RURAL EN ÁREAS DE MONTAÑA.}

La complejidad de las políticas de Desarrollo Rural no finaliza aquí. Los Estados Miembros efectúan una trasposición del acervo legislativo europeo a su propia reglamentación, con lo que se genera un amplio listado de Reglamentos, Órdenes, Reales Decretos y otras disposiciones sobre promoción de desarrollo rural. Desde la conferencia de Cork (1996) se instaba a la simplificación burocrática como herramienta para favorecer el desarrollo rural. Si bien se ha favorecido la sencillez legislativa desde la promulgación del reglamento 1257/99, todavía queda mucho por hacer para que se pueda hablar de una única política de Desarrollo rural. Siguiendo también los principios de la conferencia de Cork, se debería reforzar la territorialidad, la especificidad de las políticas de Desarrollo Rural. Esto es especialemente significativo en áreas de montaña. Se trata de áreas desfavorecidas como consecuencia de sus condiciones físicas extremas y de la vulnerabilidad de su medio natural (PUJADAS Y FONT, 1998).

El desarrollo rural debe ser en estas áreas, más que en ningún otro espacio, integrado y global. La montaña no puede ser concebida a través de acciones sectoriales que, si bien pueden llegar a generar crecimiento económico, también podrían generar al mismo tiempo importantes desequilibrios y una excesiva dependencia del exterior.

En el contexto de la Unión Europea existe preocupación por las áreas de montaña. Estas áreas han sido definidas como espacios marginales (tercer tipo de espacios rurales según el documento: El Futuro del Mundo Rural, Comisión, 1988). Los principales problemas les vienen derivados de su aislamiento geográfico, debilidad demográfica y limitado potencial de diversificación económica. Para la Unión Europea, el reto principal de estas áreas es conjugar la actividad agraria con el desarrollo de nuevas actividades. Si bien las estructuras agrarias son deficientes, la agricultura desempeña un papel fundamental, no tanto por su vertiente económica sino por las externalidades que genera (protección y conservación del paisaje).

En el documento Agenda 21, elaborado en la conferencia de Naciones Unidas sobre Medio Ambiente y Desarrollo (Río de Janeiro, 1992), el desarrollo en áreas de montaña recibe también un tratamiento específico en el capítulo 13. Este capítulo contiene dos programas específicos: A: Generación y refuerzo del 
conocimiento sobre ecología y desarrollo sostenible de los ecosistemas montanos; y B: Promoción del desarrollo integrado de cuencas y alternativas a los modos de vida actuales.

Cuadro 2. Objetivos y estrategias en el Desarrollo rural en áreas de montaña

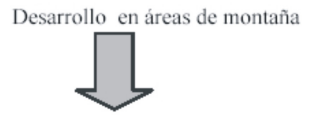

Objetivo: Mantener unos niveles de población aceptables con una calidad de vida equiparable a las zonas urbanas.
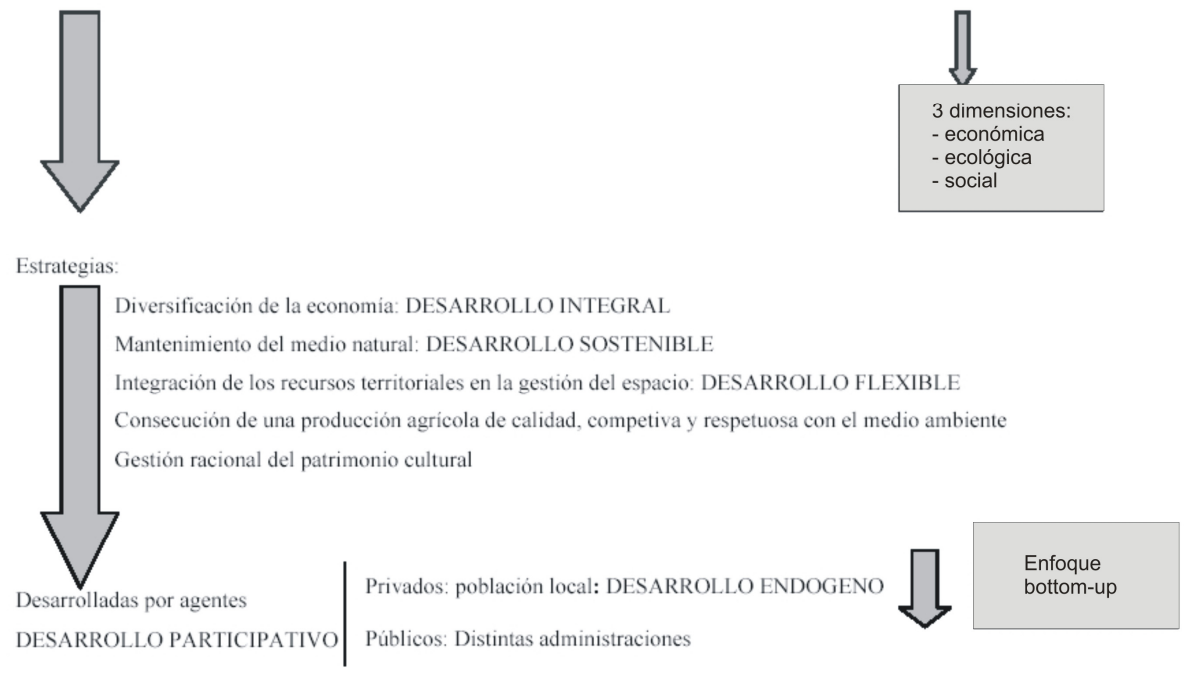

FUENTE: Elaboración propia

Los principales puntos recogidos en este capítulo de la Agenda 21 son:

- Aumento de la conciencia y del conocimiento sobre la necesidad de un desarrollo sostenible en áreas de montaña en los distintos niveles de administración (global, regional, nacional y local).

- Protección de los recursos naturales y desarrollo de mecanismos institucionales y técnicos para la reducción de las catástrofes naturales.

- Refuerzo de una red de información sobre todos aquellos temas relacionados con la montaña. 
- Combatir la pobreza a través de la implementación de actividades complementarias a la agricultura capaces de incrementar el origen de las rentas.

- Formular y negociar convenciones internacionales sobre la montaña para desarrollar un marco general de desarrollo de las áreas de montaña.

Las actuaciones sobre Desarrollo Rural y específicamente sobre Desarrollo Rural en áreas de montaña son una amalgama de políticas superpuestas sin un claro objetivo. Se hace necesaria la implantación de una política global para las áreas de montaña que busque paliar el grave problema de la despoblación y que eleve la calidad de vida de estos espacios. De esta forma se contribuirá a que sea la propia población de la montaña la que decida sobre su futuro. Una política europea de Desarrollo Rural en áreas de montaña quizá sea un proyecto demasiado ambicioso por la variedad de espacios montañosos que agrupa. Sin embargo, unas directrices generales que fuesen aplicadas subsidiariamente por cada Estado Miembro contribuirían a establecer un orden dentro de esta enjambre de reglamentos y disposiciones legislativas.

\section{Agradecimientos:}

Este trabajo ha contado con el apoyo de los proyectos de Investigación: La identificación de fuentes de sedimento y áreas generadoras de escorrentía en relación con los cambios de uso del suelo (REN 2000-1709-C04-01/G10), financiado por la CICYT, y La recuperación del espacio agrícola como estrategia de gestión integrada del territorio en áreas de montaña: el ejemplo de los altos valles del Aragón y del Gállego (PO49/2000), financiado por la DGA.

\section{BIBLIOGRAFÍA.}

ATIENZA, L. (1992): «Del proteccionismo agrario a las nuevas tendencias del desarrollo rural (estrategias de futuro)», Revista de Estudios Agrosociales, num 161, pp., 311-322

CEÑA, F. (1994): «Planteamientos económicos del desarrollo rural: Perspectiva histórica», Revista de Estudios Agrosociales, num. 169, pp., 11-52.

CHAMPETIER, Y. (1996): «El enfoque local del desarrollo rural y la iniciativa comunitaria LEADER», en VALCÁRCEL RESALT ET AL., (coord): Desarrollo local y medio ambiente. La iniciativa comunitaria LEADER, Diputación de Cuenca, 283 pp., Cuenca. 
COMISIÓn EuRoPeA. (1999): Reforma de la PAC: Desarrollo Rural, FactSheet, 11 pp., Bruselas.

GÓMEZ OREA, D. (1999): «¿Desarrollo o crisis del mundo rural?. Perspectivas de futuro», en SUÁREZ, F. Y OÑATE. J.(eds): Conservación de la naturaleza y mundo rural: experiencias y perspectivas para el siglo XXI; pp 131-150, Madrid.

LÓPEZ GÓMEZ, S. Y ARROYO ILERA, J.L.( 1987): «Las construcciones ilegales turístico-rústicas en el litoral meridional de la provincia de Valencia», en $I V$ Congreso de Geografia Agraria, pp., 73-84, Canarias.

MAYA, A. Y CABERO, V. (2000): «El desarrollo rural integrado como estrategia territorial y posible alternativa económica», Revista de economía y finanzas de Castilla y León, nº4, pp.,11-29, Madrid.

ORDOÑEZ, D. (1999): «Políticas en intervenciones de desarrollo en el medio rural», en RODRÍGUEZ GUTIÉRREZ (ED): Manual de Desarrollo Local, TREA, Oviedo, 598pp.

PARlamento Europeo. (1998): El Desarrollo Rural: Políticas Aplicadas, Situación actual del debate y perspectivas del futuro a nivel europeo, AGRI-106, Serie Agricultura, Montes y Desarrollo Rural, 264pp., Luxemburgo.

PARLAMENTO EUROPEO (2001): Fichas técnicas. www.europarl.eu

PUJADAS, R.Y FONT, J. (1998): Ordenación y planificación territorial, Espacios y Sociedades, serie mayor, Síntesis, Madrid, 399pp.

RAMOS, E. Y ROMERO, J. (1995): «Para una concepción sistémica del desarrollo rural», en RAMOS, E. Y CRUZ, J. (coord.): Hacia un nuevo sistema rural, MAPA, Serie Estudios, Madrid: 49-90.

USERO, L. (1998): La Unión Europea y el Desarrollo Rural, Federación Aragonesa de Municipios y Provincias, Real Instituto de Estudios Europeos, Zaragoza.

VALCÁRCEL RESALT, G. (1989): «El desarrollo local en zonas desfavorecidas españolas», Revista de Estudios Agrosociales, Num.149, pp., 193-235.

VICENTE SERRANO, S. (2000): Efectos en la gestión de los recursos de los diferentes sistemas ganaderos de montaña: El caso del valle de Borau, Tesis de Licenciatura, Zaragoza, $367 \mathrm{pp}$.

ZIMMERMAN, F. (1994): «El papel del turismo en la reestructuración de las regiones alpinas». Papers de Turisme 16: 77-87 